Research Article

\title{
Noncommutative Correction to the Entropy of BTZ Black Hole with GUP
}

\author{
M. A. Anacleto $\mathbb{D},{ }^{1}$ F. A. Brito $\mathbb{D},,^{1,2}$ B. R. Carvalho, ${ }^{1}$ and E. Passos $\mathbb{D}^{1}$ \\ ${ }^{1}$ Departamento de Física, Universidade Federal de Campina Grande, Caixa Postal 10071, 58429-900 Campina Grande, \\ Paraíba, Brazil \\ ${ }^{2}$ Departamento de Física, Universidade Federal da Paraíba, Caixa Postal 5008, 58051-970 João Pessoa, Paraíba, Brazil
}

Correspondence should be addressed to F. A. Brito; fabrito@df.ufcg.edu.br

Received 22 October 2020; Revised 18 December 2020; Accepted 28 December 2020; Published 12 January 2021

Academic Editor: Hasan El moumni

Copyright (C) 2021 M. A. Anacleto et al. This is an open access article distributed under the Creative Commons Attribution License, which permits unrestricted use, distribution, and reproduction in any medium, provided the original work is properly cited. The publication of this article was funded by $\mathrm{SCOAP}^{3}$.

We investigate the effect of noncommutativity and quantum corrections to the temperature and entropy of a BTZ black hole based on a Lorentzian distribution with the generalized uncertainty principle (GUP). To determine the Hawking radiation in the tunneling formalism, we apply the Hamilton-Jacobi method by using the Wentzel-Kramers-Brillouin (WKB) approach. In the present study, we have obtained logarithmic corrections to entropy due to the effect of noncommutativity and GUP. We also address the issue concerning stability of the noncommutative BTZ black hole by investigating its modified specific heat capacity.

\section{Introduction}

The study of three-dimensional gravity has been extensively explored in the literature [1]. It has become an excellent laboratory for a better understanding of the fundamentals of classical and quantum gravity and also to explore some ideas behind the AdS/CFT correspondence [2]. This special attention in three-dimensional gravity has been mainly due to the discovery of the black hole solution in $2+1$ dimensions [3]. In addition, generalizations of the Bañados-TeitelboimZanelli (BTZ) black hole solution have also been constructed considering coupling with a dilaton/scalar field [4-6]. In recent years, the implementation of noncommutativity in black hole physics has been extensively explored (for a review see [7]). In [8], the authors have introduced a noncommutative Schwarzschild black hole solution in four dimensions. As shown in [8], one way to incorporate noncommutativity into general relativity is to modify the source of matter. Thus, noncommutativity is introduced by replacing the point-like source term with a Gaussian distribution - or otherwise by a Lorentzian distribution [9]. In addition, noncommutativity in a BTZ black hole has also been introduced in [10-14]. In [15], the gravitational Aharonov-Bohm effect due to a BTZ black hole in a noncommutative background has been analyzed. The process of massless scalar wave scattering by a noncommutative black hole via a Lorentzian smeared mass distribution has been explored in [16]. The thermodynamic properties of BTZ black holes in noncommutative spaces have been studied in [17-20].

It is well known that string theories, loop quantum gravity, and noncommutative geometry presents important elements for the construction of a compatible theory of quantum gravity. Furthermore, these theories have a common feature, which is the appearance of a minimum length on the order of the Planck scale. This therefore leads to a modification of the Heisenberg uncertainty principle, which is called the generalized uncertainty principle (GUP) [21-23]. In recent years, several works have been devoted to investigating the effect of GUP on computing the Hawking radiation from black holes in $2+1$ dimensions. In this sense, the HamiltonJacobi method via the WKB approach to calculate the imaginary part of the action is an effective way of investigating the Hawking radiation as a process of tunneling particles from a black hole [24-30]. In [31], the effect of GUP on the Hawking radiation from the BTZ black hole has been investigated using the modified Dirac equation. The Hawking radiation 
has been analyzed in [32], by considering the MartinezZanelli black hole in $2+1$ dimensions [5] and using the Dirac equation modified by the GUP. By applying the quantum tunneling formalism, the Hawking radiation from a new type of black hole in $2+1$ dimensions has also been studied in [33], and in [34], the Hawking radiation of a charged rotating BTZ black hole with GUP was explored. Moreover, in $[35,36]$, the entropy of the BTZ black hole with GUP has been determined, and in [37], by adopting a new principle of extended uncertainty, its effect on the thermodynamics of the black hole has been examined.

The purpose of this paper is to investigate the effect of noncommutative and quantum corrections coming from GUP for the calculation of the temperature and entropy of a BTZ black hole based on a Lorentzian distribution, by considering the tunneling formalism framework through the Hamilton-Jacobi method. Thus, the Hawking radiation will be computed using the WKB approach. Therefore, we show that the entropy of the BTZ black hole presents logarithmic corrections due to both of the aforementioned effects.

The paper is organized as follows. In Section 2, we consider noncommutative corrections for the BTZ black hole metric implemented via the Lorentzian mass distribution. We also have applied the Hamilton-Jacobi approach to determine noncommutative corrections for the Hawking temperature and entropy. In Section 3, we consider GUP to compute quantum corrections to the Hawking temperature and entropy and also briefly comment on the correction of the specific heat capacity at a constant volume. In Section 4, we make our final considerations.

\section{Noncommutative Corrections to the BTZ Black Holes}

Here, we introduce the noncomutativity by considering a Lorentzian mass distribution, given by $[8,9,14,16]$ :

$$
\rho_{\theta}(r)=\frac{M \sqrt{\theta}}{2 \pi\left(r^{2}+\theta\right)^{3 / 2}}
$$

where $\theta$ is the noncommutative parameter with dimension of length ${ }^{2}$ and $M$ is the total mass diffused throughout the region of linear size $\sqrt{\theta}$. In this case, the smeared mass distribution function becomes [14]

$$
\begin{aligned}
M_{\theta} & =\int_{0}^{r} \rho_{\theta}(r) 2 \pi r d r=M\left(1-\frac{\sqrt{\theta}}{\sqrt{r^{2}+\theta}}\right) \\
& =M-\frac{M \sqrt{\theta}}{r}+\mathcal{O}\left(\theta^{3 / 2}\right) .
\end{aligned}
$$

By considering the above modified mass, the metric of the noncommutative BTZ black hole is given by

$$
d s^{2}=-\mathscr{F}(r) d t^{2}+\mathscr{F}(r)^{-1} d r^{2}+r^{2}\left(d \phi-\frac{J}{2 r^{2}} d t\right)^{2}
$$

where

$$
\mathscr{F}(r)=-M_{\theta}+\frac{r^{2}}{l^{2}}+\frac{J^{2}}{4 r^{2}}=-M+\frac{M \sqrt{\theta}}{r}+\frac{r^{2}}{l^{2}}+\frac{J^{2}}{4 r^{2}} .
$$

Note that the metric obtained by a noncommutative correction is different from the metric in [1]. A term, $M \sqrt{\theta} / r$, of the Schwarzschild type is generated due to the noncommutative correction. Our metric shows similarities with the metric obtained in $[4,5]$ and also with one of the classes of solutions found in [6] with a dilaton/scalar field.

We shall now analyze the nonrotating case $(J=0)$, so the metric (3) becomes

$$
d s^{2}=-f(r) d t^{2}+f(r)^{-1} d r^{2}+r^{2} d \phi^{2}
$$

where

$$
f(r)=-M+\frac{M \sqrt{\theta}}{r}+\frac{r^{2}}{l^{2}}
$$

The horizons are found by solving

$$
f(r)=-M+\frac{M \sqrt{\theta}}{r}+\frac{r^{2}}{l^{2}}=0
$$

which is equivalent to solving a cubic equation

$$
r^{3}-M l^{2} r+M l^{2} \sqrt{\theta}=0 .
$$

The roots of this cubic equation are given by [38]:

$$
r=2 \sqrt{\frac{l^{2} M}{3}} \sin \left[\frac{1}{3} \sin ^{-1}\left(\frac{3}{2} \sqrt{\frac{3 \theta}{l^{2} M}}\right)+\varepsilon \frac{2 \pi}{3}\right], \quad \varepsilon \in\{0, \pm 1\} .
$$

The three roots for $\varepsilon=1,0,-1$, up to the first order in $\sqrt{\theta}$, are given, respectively, by

$$
\begin{aligned}
& \tilde{r}_{h}=r_{h}-\frac{\sqrt{\theta}}{2}+\cdots, \\
& r_{c}=\sqrt{\theta}+\cdots, \\
& r_{v}=-r_{h}-\frac{\sqrt{\theta}}{2}+\cdots,
\end{aligned}
$$

where $r_{h}=\sqrt{l^{2} M}, \tilde{r}_{h}$ is the event horizon, $r_{c}$ the cosmological horizon, and $r_{v}$ the virtual (unphysical) horizon. From equation (7), we obtain the mass of the noncommutative black hole, up to the first order in $\sqrt{\theta}$, that is given by

$$
M=\frac{\tilde{r}_{h}^{2}}{l^{2}}+\frac{\tilde{r}_{h} \sqrt{\theta}}{l^{2}}+\cdots .
$$


In order to compute the Hawking temperature, we use the Klein-Gordon equation for a scalar field $\Phi$ in the curved space given by

$$
\left[\frac{1}{\sqrt{-g}} \partial_{\mu}\left(\sqrt{-g} g^{\mu v} \partial_{v}\right)-\frac{m^{2}}{\hbar^{2}}\right] \Phi=0
$$

where $m$ is the mass of a scalar particle. In the sequel, we apply the WKB approximation

$$
\Phi=\exp \left[\frac{i}{\hbar} I\left(t, r, x^{i}\right)\right]
$$

such that we obtain

$$
g^{\mu \nu} \partial_{\mu} I \partial_{v} I+m^{2}=0
$$

By applying the metric (5) in the above equation, we have

$$
-\frac{1}{f(r)}\left(\partial_{t} I\right)^{2}+f(r)\left(\partial_{r} I\right)^{2}+\frac{1}{r^{2}}\left(\partial_{\phi} I\right)^{2}+m^{2}=0
$$

Now, we can write the solution of equation (15) as follows:

$$
I=-E t+W(r)+J_{\phi} \phi
$$

where

$$
\begin{aligned}
& \partial_{t} I=-E, \\
& \partial_{r} I=\frac{d W(r)}{d r}, \\
& \partial_{\phi} I=J_{\phi},
\end{aligned}
$$

with $J_{\phi}$ being a constant. By substituting (16) into equation (15) and solving for $W(r)$, the classical action is written as follows:

$$
I=-E t+\int d r \frac{\sqrt{E^{2}-f(r)\left(\left(J_{\phi}^{2} / r^{2}\right)+m^{2}\right)}}{f(r)}+J_{\phi} \phi .
$$

Next, in the regime near the event horizon of the noncommutative BTZ black hole, $r \longrightarrow \tilde{r}_{h}$, we can write $f(r) \approx \kappa\left(r-\tilde{r}_{h}\right)$, and so the spatial part of the action function reads

$$
W(r)=\frac{1}{\kappa} \int d r \frac{\sqrt{E^{2}-\kappa\left(r-\tilde{r}_{h}\right)\left(\left(J_{\phi}^{2} / r^{2}\right)+m^{2}\right)}}{\left(r-\tilde{r}_{h}\right)}=\frac{2 \pi i}{\kappa} E,
$$

where $\kappa$ is the surface gravity of the noncommutative BTZ black hole given by

$$
\kappa=f^{\prime}\left(\tilde{r}_{h}\right)=\frac{2 \tilde{r}_{h}}{l^{2}}-\frac{M \sqrt{\theta}}{\tilde{r}_{h}^{2}} .
$$

The next step is to determine the probability of tunneling for a particle with energy $E$, and for this, we use the following expression:

$$
\Gamma \simeq \exp [-2 \operatorname{Im}(\mathrm{I})]=\exp \left(-\frac{4 \pi E}{\kappa}\right)
$$

In order to calculate the Hawking temperature of the noncommutative BTZ black hole, we can compare equation (21) with the Boltzmann factorexp $\left(-E / \widetilde{T}_{H}\right)$, so we can find

$$
\tilde{T}_{H}=\frac{\kappa}{4 \pi}=\frac{\tilde{r}_{h}}{2 \pi l^{2}}-\frac{M \sqrt{\theta}}{4 \pi \tilde{r}_{h}^{2}}
$$

Moreover, the above result can be rewritten in terms of $r_{h}=\sqrt{l^{2} M}$ as follows:

$$
\tilde{T}_{H}=\frac{r_{h}-\sqrt{\theta} / 2}{2 \pi l^{2}}-\frac{M \sqrt{\theta}}{4 \pi r_{h}^{2}}=T_{h}-\frac{\sqrt{\theta}}{4 \pi l^{2}}-\frac{M \sqrt{\theta}}{4 \pi r_{h}^{2}} .
$$

Therefore, the result above shows that the Hawking temperature is modified due to the presence of the noncommutative parameter $\theta$. Note that when we take $\theta=0$, we recover the temperature of the commutative BTZ black hole, which is $T_{h}=r_{h} /\left(2 \pi l^{2}\right)$.

At this point, we are prepared to go further. Let us now consider the noncommutative BTZ black hole in the rotating regime $(J \neq 0)$. Now the line element of equation (3) can be written in the form

$$
d s^{2}=-\mathscr{F}(r) d t^{2}+\mathscr{F}(r)^{-1} d r^{2}+r^{2} d \varphi^{2},
$$

where

$$
\begin{aligned}
\mathscr{F}(r) & =-M+\frac{M \sqrt{\theta}}{r}+\frac{r^{2}}{l^{2}}+\frac{J^{2}}{4 r^{2}}, \\
d \varphi & =d \phi-\frac{J}{2 r^{2}} d t .
\end{aligned}
$$

Thus, to find the horizons, we have to solve

$$
\mathscr{F}(r)=-M+\frac{M \sqrt{\theta}}{r}+\frac{r^{2}}{l^{2}}+\frac{J^{2}}{4 r^{2}}=0,
$$

which is equivalent to solving a quartic equation

$$
r^{4}-l^{2} M r^{2}+\frac{l^{2} J^{2}}{4}+l^{2} M \sqrt{\theta} r=0 .
$$


We can now rewrite this equation as follows [38]:

$$
\left(r^{2}-r_{+}^{2}\right)\left(r^{2}-r_{-}^{2}\right)+l^{2} M \sqrt{\theta} r=0,
$$

that for $\theta=0$, we have

$$
r_{ \pm}^{2}=\frac{l^{2} M}{2}\left[1 \pm \sqrt{1-\left(\frac{J}{M l}\right)^{2}}\right]
$$

where $r_{+}$is the outer event horizon and $r_{-}$is the inner event horizon of the commutative BTZ black hole. Now, rearranging equation (28) in the form

$$
r^{2}=r_{ \pm}^{2}-\frac{r_{h}^{2} \sqrt{\theta} r}{r^{2}-r_{\mp}^{2}}
$$

where $r_{h}=\sqrt{l^{2} M}$, we can solve it perturbatively. So, in the first approximation we get the event horizon:

$$
\tilde{r}_{+}^{2} \approx r_{+}^{2}+\frac{r_{h}^{2} \sqrt{\theta} r_{+}}{r_{-}^{2}-r_{+}^{2}}
$$

or by keeping terms up to the first order in $\sqrt{\theta}$, we obtain

$$
\tilde{r}_{+}=r_{+}+\frac{r_{h}^{2} \sqrt{\theta}}{2\left(r_{-}^{2}-r_{+}^{2}\right)}+\cdots,
$$

for the outer horizon. For the internal horizon, we have

$$
\tilde{r}_{-}^{2} \approx r_{-}^{2}-\frac{r_{h}^{2} \sqrt{\theta} r_{-}}{r_{-}^{2}-r_{+}^{2}}
$$

so that for $\tilde{r}_{-}$, we find

$$
\tilde{r}_{-}=r_{-}-\frac{r_{h}^{2} \sqrt{\theta}}{2\left(r_{-}^{2}-r_{+}^{2}\right)}+\cdots
$$

In order to determine the Hawking temperature for the case of the rotating black hole, we can follow the same steps as presented above, and so for the tunneling probability we have

$$
\Gamma=\exp \left[-\frac{4 \pi E}{\bar{\kappa}}\right]
$$

where the surface gravity is given by

$$
\bar{\kappa}=\mathscr{F}^{\prime}\left(\tilde{r}_{+}\right)=\frac{2 \tilde{r}_{+}}{l^{2}}\left(1-\frac{l^{2} J^{2}}{4 \tilde{r}_{+}^{4}}\right)-\frac{M \sqrt{\theta}}{\tilde{r}_{+}^{2}} .
$$

Again, by comparing $\Gamma$ with the Boltzmann factor $\exp \left(-E / \mathscr{T}_{H}\right)$, we obtain the Hawking temperature of the noncommutative rotating BTZ black hole:

$$
\mathscr{T}_{H}=\frac{\bar{\kappa}}{4 \pi}=\frac{\mathscr{F}^{\prime}\left(\tilde{r}_{+}\right)}{4 \pi}=\frac{2 \tilde{r}_{+}}{4 \pi l^{2}}\left(1-\frac{l^{2} J^{2}}{4 \tilde{r}_{+}^{4}}\right)-\frac{M \sqrt{\theta}}{4 \pi \tilde{r}_{+}^{2}} .
$$

For $\theta=0$, we recover the result for the Hawking temperature of the rotating BTZ black hole which is given by

$$
\mathscr{T}_{h}=\frac{r_{+}}{2 \pi l^{2}}\left(1-\frac{l^{2} J^{2}}{4 r_{+}^{4}}\right) .
$$

From equation (26), we obtain the mass of the noncommutative black hole, up to the first order in $\sqrt{\theta}$, that is given by

$$
M=\frac{\tilde{r}_{+}^{2}}{l^{2}}+\frac{J^{2}}{4 \tilde{r}_{+}^{2}}+\frac{\tilde{r}_{+} \sqrt{\theta}}{l^{2}}+\frac{\sqrt{\theta} J^{2}}{4 \tilde{r}_{+}^{3}}+\cdots .
$$

In order to analyze the entropy, we consider the following equation:

$$
S=\int \frac{1}{\widetilde{T}_{H}} \frac{\partial M}{\partial \tilde{r}_{+}} d \tilde{r}_{+}
$$

where

$$
\frac{\partial M}{\partial \tilde{r}_{+}}=\frac{2 \tilde{r}_{+}}{l^{2}}\left(1-\frac{l^{2} J^{2}}{4 \tilde{r}_{+}^{4}}\right)+\frac{\sqrt{\theta}}{l^{2}}\left(1-\frac{3 l^{2} J^{2}}{4 \tilde{r}_{+}^{4}}\right)+\cdots
$$

The next step is to perform an expansion in $\mathscr{T}_{H}^{-1}$ up to the first order in $\sqrt{\theta}$, so we can find

$$
\mathscr{T}_{H}^{-1}=4 \pi\left[\frac{2 \tilde{r}_{+}}{l^{2}}\left(1-\frac{l^{2} J^{2}}{4 \tilde{r}_{+}^{4}}\right)\right]^{-1}\left\{1+\frac{r_{h}^{2} \sqrt{\theta}}{2 \tilde{r}_{+}^{3}}\left(1-\frac{l^{2} J^{2}}{4 \tilde{r}_{+}^{4}}\right)^{-1}\right\}+\cdots
$$

Now, by replacing (41) and (42) in (40), we obtain

$$
\begin{aligned}
\widehat{S}= & 4 \pi \int\left\{1+\left[\frac{r_{h}^{2} \sqrt{\theta}}{2 \tilde{r}_{+}^{3}}+\frac{\sqrt{\theta}}{2 \tilde{r}_{+}}\left(1-\frac{3 l^{2} J^{2}}{4 \tilde{r}_{+}^{4}}\right)\right]\left(1+\frac{l^{2} J^{2}}{4 \tilde{r}_{+}^{4}}\right)+\cdots\right\} d \tilde{r}_{+} \\
= & 4 \pi \tilde{r}_{+}+2 \pi \sqrt{\theta} \ln \left(\tilde{r}_{+}\right)-\frac{\pi r_{h}^{2} \sqrt{\theta}}{\tilde{r}_{+}^{2}}-\frac{\pi r_{h}^{2} l^{2} J^{2} \sqrt{\theta}}{12 \tilde{r}_{+}^{6}}+\frac{2 \pi l^{2} J^{2} \sqrt{\theta}}{8 \tilde{r}_{+}^{4}} \\
& +\frac{3 \pi l^{4} J^{4} \sqrt{\theta}}{64 \tilde{r}_{+}^{8}}+S_{0}+\cdots,
\end{aligned}
$$


where $S_{0}$ is an integration constant, and by rewriting in terms of $r_{+}$, we have

$$
\begin{aligned}
\widehat{S}= & 4 \pi r_{+}+\frac{2 \pi r_{h}^{2} \sqrt{\theta}}{\left(r_{-}^{2}-r_{+}^{2}\right)}+2 \pi \sqrt{\theta} \ln \left(r_{+}\right)-\frac{\pi r_{h}^{2} \sqrt{\theta}}{r_{+}^{2}}-\frac{\pi r_{h}^{2} l^{2} J^{2} \sqrt{\theta}}{12 r_{+}^{6}} \\
& +\frac{2 \pi l^{2} J^{2} \sqrt{\theta}}{8 r_{+}^{4}}+\frac{3 \pi l^{4} J^{4} \sqrt{\theta}}{64 r_{+}^{8}}+S_{0}+\cdots
\end{aligned}
$$

For $\theta=0$ in (44), we have $S=4 \pi r_{+}$, which is the entropy of the commutative rotating BTZ black hole. On the other hand, for the case $J=0$, we have $r_{+}=r_{h}$, and the entropy becomes

$$
\widehat{S}=4 \pi\left(r_{h}-\frac{3 \sqrt{\theta}}{2}\right)+3 \pi \sqrt{\theta}+2 \pi \sqrt{\theta} \ln \left(r_{h}\right)+S_{0}+\cdots
$$

Note that we have obtained a logarithmic correction for the noncommutative BTZ black hole. Besides, our metric corresponds to that of Ref. [6] with the equivalence $\sqrt{\theta} \equiv B$ and which is given for the nonrotating case $(J=0)$ by

$$
f_{B}(r)=-M+\frac{M B}{r}+\frac{r^{2}}{l^{2}}
$$

where $B$ is a finite constant parameter introduced by a dilaton/scalar field. Hence, the horizon radius can be computed from equation (9) as above by taking the approximation $B / \sqrt{l^{2} M}=B \sqrt{\Lambda / M} \ll 1$. So, we find

$$
r_{h b}=r_{h}-\frac{B}{2}+\cdots
$$

Thus, from equation (40), a logarithmic correction is obtained for entropy, given by

$$
S_{B}=4 \pi r_{h b}+2 \pi B \ln \left(r_{h b}\right)+\cdots,
$$

with $\Delta S \equiv S_{B}-S=2 \pi B \ln r_{h}+\cdots \quad$ associated with small (thermal) fluctuations. This approach could also be considered in [2-5].

\section{Quantum Correction to the Entropy}

In this section, in order to derive quantum corrections to the Hawking temperature and entropy of the noncommutative BTZ black hole, we will apply tunneling formalism using the Hamilton-Jacobi method. So, we will adopt the following GUP [39], [40-49]:

$$
\Delta x \Delta p \geq \frac{\hbar}{2}\left(1-\frac{\alpha l_{p}}{\hbar} \Delta p+\frac{\alpha^{2} l_{p}^{2}}{\hbar^{2}}(\Delta p)^{2}\right)
$$

where $\alpha$ is a dimensionless positive parameter and $l_{p}$ is the Planck length.
In sequence, without loss of generality, we will adopt the natural units $G=c=k_{B}=\hbar=l_{p}=1$, and by assuming that $\Delta p \sim E$ and following the steps performed in [24], we can obtain the following relation for the corrected energy of the black hole:

$$
E_{\text {gup }} \geq E\left[1-\frac{\alpha}{2(\Delta x)}+\frac{\alpha^{2}}{2(\Delta x)^{2}}+\cdots\right]
$$

Thus, performing the same procedure as previously described, we have the following result for the probability of tunneling with corrected energy $E_{\text {gup }}$ given by

$$
\Gamma \simeq \exp [-2 \operatorname{I} m(I)]=\exp \left[\frac{-4 \pi E_{\text {gup }}}{a}\right]
$$

where $a$ is the surface gravity. Again, we compare with the Boltzmann factor and we obtain the corrected Hawking temperature of the noncommutative BTZ black hole

$$
T \leq \tilde{T}_{H}\left[1-\frac{\alpha}{2(\Delta x)}+\frac{\alpha^{2}}{2(\Delta x)^{2}}+\cdots\right]^{-1}
$$

Here, for simplicity, we will consider the case $J=0$. The temperature $\tilde{T}_{H}$ is given by equation (22). Furthermore, since near the event horizon of the BTZ black hole the minimum uncertainty in our model is of the order of the radius of the horizon, so the corrected temperature due to the GUP is given by

$$
\begin{aligned}
T_{\text {gup }} & \leq \tilde{T}_{H}\left(1-\frac{\alpha}{4 \tilde{r}_{h}}+\frac{\alpha^{2}}{8 \tilde{r}_{h}^{2}}+\cdots\right)^{-1} \\
& =\frac{2 \tilde{r}_{h}}{4 \pi l^{2}}\left(1-\frac{r_{h}^{2} \sqrt{\theta}}{2 \tilde{r}_{h}^{3}}\right)\left(1+\frac{\alpha}{4 \tilde{r}_{h}}-\frac{\alpha^{2}}{8 \tilde{r}_{h}^{2}}+\cdots\right) .
\end{aligned}
$$

We can also write the result above in terms of $r_{h}=l$ $\sqrt{M}$ as follows:

$$
\begin{aligned}
T_{\text {gup }} \leq & \frac{2 r_{h}}{4 \pi l^{2}}\left(1-\frac{\sqrt{\theta}}{2 r_{h}}\right)^{2}\left[1+\frac{\alpha}{4 r_{h}}\left(1+\frac{\sqrt{\theta}}{2 r_{h}}+\cdots\right)\right. \\
& \left.-\frac{\alpha^{2}}{8 r_{h}^{2}}\left(1+\frac{\sqrt{\theta}}{r_{h}}+\cdots\right)+\cdots\right] .
\end{aligned}
$$

Next, we will compute the entropy of the noncommutative BTZ black hole by using the following formula:

$$
S_{\text {gup }}=\int \frac{1}{T_{\text {gup }}} \frac{\partial M}{\partial \tilde{r}_{h}} d \tilde{r}_{h}
$$


where from equation (11), we have

$$
\frac{\partial M}{\partial \tilde{r}_{h}}=\frac{2 \tilde{r}_{h}}{l^{2}}\left(1+\frac{\sqrt{\theta}}{2 \tilde{r}_{h}}\right)
$$

So, now we can obtain the corrected entropy

$$
\begin{aligned}
S_{\text {gup }}= & 4 \pi \int\left(1+\frac{\sqrt{\theta}}{2 \tilde{r}_{h}}\right)\left(1+\frac{r_{h}^{2} \sqrt{\theta}}{2 \tilde{r}_{h}^{3}}\right)\left[1-\frac{\alpha}{4 \tilde{r}_{h}}+\frac{\alpha^{2}}{8 \tilde{r}_{h}^{2}}+\cdots\right] d \tilde{r}_{h} \\
= & 4 \pi \tilde{r}_{h}+2 \pi \sqrt{\theta} \ln \left(\tilde{r}_{h}\right)-\frac{\pi r_{h}^{2} \sqrt{\theta}}{\tilde{r}_{h}^{2}}-\pi \alpha \ln \left(\tilde{r}_{h}\right)-\frac{\pi \alpha^{2}}{2 \tilde{r}_{h}} \\
& +\frac{\pi \alpha \sqrt{\theta}}{2 \tilde{r}_{h}}-\frac{\pi \alpha^{2} \sqrt{\theta}}{8 \tilde{r}_{h}^{2}}+\frac{\pi r_{h}^{2} \alpha \sqrt{\theta}}{6 \tilde{r}_{h}^{3}}-\frac{\pi r_{h}^{2} \alpha^{2} \sqrt{\theta}}{16 \tilde{r}_{h}^{4}}+S_{0}+\cdots,
\end{aligned}
$$

or by expressing the result above in terms of the $r_{h}$ we have

$$
\begin{aligned}
S_{\text {gup }}= & 4 \pi\left(r_{h}-\frac{3 \sqrt{\theta}}{2}\right)+2 \pi \sqrt{\theta} \ln \left(r_{h}\right)+3 \pi \sqrt{\theta}-\pi \alpha \ln \left(r_{h}\right) \\
& -\frac{\pi \alpha^{2}}{2 r_{h}}+\frac{2 \pi \alpha \sqrt{\theta}}{3 r_{h}}-\frac{3 \pi \alpha^{2} \sqrt{\theta}}{16 r_{h}^{2}}+S_{0}+\cdots .
\end{aligned}
$$

Therefore, by analyzing the result, we have obtained corrections to the entropy due to the effects of GUP and also noncommutative correction. Note that due to the effect of noncommutativity and GUP, we have found logarithmic corrections for the entropy of the BTZ black hole. For $\alpha=0$, we have precisely the noncommutative correction to the entropy given by (45). In [50], the authors analyzed the thermodynamics of the charged rotating BTZ black hole, and logarithmic corrections were also obtained for entropy in the presence of the GUP and thermal fluctuations (for small variations in $\beta=1 / T)$. The logarithmic corrections to entropy become important for very small black holes and negligible for very large black holes. Further studies addressing these issues were also considered in Refs. [51-54]. In our case, logarithmic corrections are due to the presence of GUP and/or the noncommutativity of spacetime that mimic small thermal fluctuations by properly identifying the corresponding parameter to values normally found in thermal fluctuations as well discussed in Refs [50-54].

At this point, we will compute the Helmholtz free energy, which can be determined by using the following relationship:

$$
F_{\text {gup }}=-\int S_{\text {gup }} d T_{\text {gup }}
$$

So, from equations (53) and (57), we get

$$
\begin{aligned}
F_{\text {gup }}= & -\frac{1}{2 \pi l^{2}} \int\left(1+\frac{r_{h}^{2} \sqrt{\theta}}{\tilde{r}_{h}^{3}}+\frac{\alpha^{2}}{8 \tilde{r}_{h}^{2}}+\frac{3 r_{h}^{2} \alpha \sqrt{\theta}}{8 \tilde{r}_{h}^{4}}-\frac{r_{h}^{2} \alpha^{2} \sqrt{\theta}}{4 \tilde{r}_{h}^{5}}\right) S_{\text {gup }} d \tilde{r}_{h} \\
= & -\frac{\tilde{r}_{h}^{2}}{l^{2}}+\frac{3 r_{h}^{2} \sqrt{\theta}}{2 l^{2} \tilde{r}_{h}}+\frac{5 r_{h}^{2} \alpha \sqrt{\theta}}{12 l^{2} \tilde{r}_{h}^{2}}-\frac{29 r_{h}^{2} \alpha^{2} \sqrt{\theta}}{96 l^{2} \tilde{r}_{h}^{3}}-\frac{\sqrt{\theta} \tilde{r}_{h}}{l^{2}} \ln \left(\tilde{r}_{h}\right) \\
& +\frac{\sqrt{\theta} \tilde{r}_{h}}{l^{2}}+\frac{\alpha^{2} \sqrt{\theta} \ln \left(\tilde{r}_{h}\right)}{8 l^{2} \tilde{r}_{h}}+\frac{\alpha^{2} \sqrt{\theta}}{16 l^{2} \tilde{r}_{h}}+\frac{\alpha \tilde{r}_{h}}{2 l^{2}} \ln \left(\tilde{r}_{h}\right)-\frac{\alpha \tilde{r}_{h}}{2 l^{2}} \\
& -\frac{r_{h}^{2} \alpha^{2} \sqrt{\theta} \ln \left(\tilde{r}_{h}\right)}{16 l^{2} \tilde{r}_{h}^{3}}-\frac{\alpha \sqrt{\theta} \ln \left(\tilde{r}_{h}\right)}{4 l^{2}}+F_{0}+\cdots,
\end{aligned}
$$

where $F_{0}$ is an integration constant.

For $\alpha=0$ (in the absence of the GUP), the Helmholtz free energy becomes

$$
F_{\theta}=-\frac{\tilde{r}_{h}}{l^{2}}\left(\tilde{r}_{h}-\sqrt{\theta}\right)+\frac{3 r_{h}^{2} \sqrt{\theta}}{2 l^{2} \tilde{r}_{h}}-\frac{\sqrt{\theta} \tilde{r}_{h}}{l^{2}} \ln \left(\tilde{r}_{h}\right)+F_{0}+\cdots,
$$

or rewriting in terms of $r_{h}$, we have

$$
\begin{aligned}
F_{\theta}= & -\frac{1}{l^{2}}\left(r_{h}-\frac{\sqrt{\theta}}{2}\right)\left(r_{h}-\frac{3 \sqrt{\theta}}{2}\right)+\frac{3 r_{h} \sqrt{\theta}}{2 l^{2}} \\
& -\frac{\sqrt{\theta} r_{h}}{l^{2}} \ln \left(r_{h}\right)+F_{0}+\cdots
\end{aligned}
$$

The correction of the specific heat capacity is given by

$$
\begin{aligned}
C_{\text {gup }} & =\frac{\partial M}{\partial T_{\text {gup }}}=\frac{\partial M}{\partial \tilde{r}_{h}}\left(\frac{\partial T_{\text {gup }}}{\partial \tilde{r}_{h}}\right)^{-1} \\
& =4 \pi \tilde{r}_{h}\left(1+\frac{\sqrt{\theta}}{2 \tilde{r}_{h}}\right)\left(1-\frac{\sqrt{\theta}}{\tilde{r}_{h}}-\frac{\alpha^{2}}{8 \tilde{r}_{h}^{2}}-\frac{3 \alpha \sqrt{\theta}}{8 \tilde{r}_{h}^{2}}+\frac{\alpha^{2} \sqrt{\theta}}{4 \tilde{r}_{h}^{3}}+\cdots\right)
\end{aligned}
$$

Now if $\alpha=0$ in equation (63), we have

$$
C_{\theta}=4 \pi \tilde{r}_{h}\left(1+\frac{\sqrt{\theta}}{2 \tilde{r}_{h}}\right)\left(1-\frac{\sqrt{\theta}}{\tilde{r}_{h}}\right)+\cdots
$$

which in terms of $r_{h}$, becomes

$$
C_{\theta}=4 \pi r_{h}\left(1+\frac{\sqrt{\theta}}{2 r_{h}}\right)\left(1-\frac{3 \sqrt{\theta}}{2 r_{h}}\right)+\cdots
$$

For $\theta=0$, we have $C=4 \pi r_{h}$, which is the specific heat for the commutative BTZ black hole. Note that the specific heat 


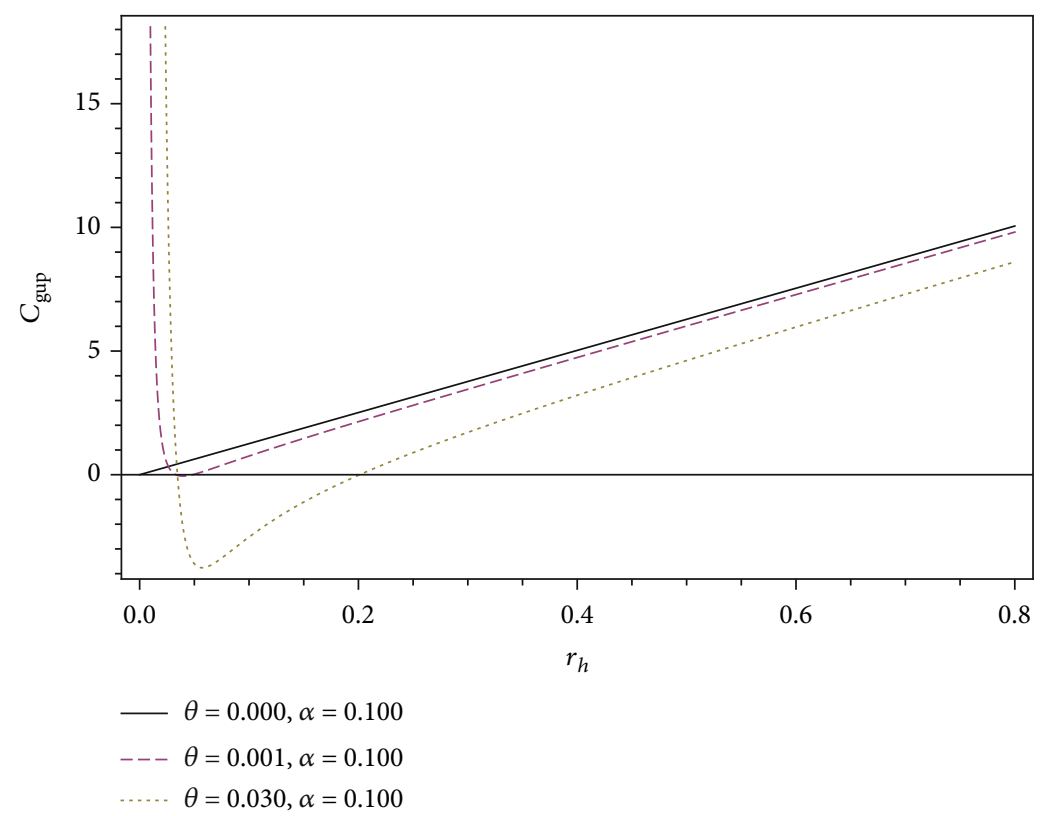

FIGURE 1: Specific heat capacity (equation (63)). For $\theta=\alpha=0$, we have the result for the specific heat of the commutative BTZ black hole. We also show the result when $\theta \neq 0$ and $\alpha \neq 0$.

vanishes at the point $r_{h}=3 \sqrt{\theta} / 2$ (or $\tilde{r}_{h}=\sqrt{\theta}$ in equation (64)). In this case, we have a minimum radius

$$
r_{\theta \min }=\sqrt{l^{2} M_{\theta \min }}=\frac{3 \sqrt{\theta}}{2},
$$

and then the noncommutative black hole reaches a minimum mass given by

$$
M_{\theta \min }=\frac{9 \theta}{4 l^{2}} .
$$

Thus, this result indicates that the black hole ceases to evaporate completely and becomes a remnant. Next, we obtain the temperature of the remnant of the black hole by replacing the $r_{h} \longrightarrow r_{\theta \min }$ in (23):

$$
T_{\theta \mathrm{rem}} \approx \frac{\tilde{T}_{H}}{3}=\frac{\mathrm{r}_{\theta \mathrm{min}}}{6 \pi l^{2}}=\frac{\sqrt{\theta}}{4 \pi l^{2}} .
$$

Furthermore, from equations (45) and (62) for $r_{h} \longrightarrow$ $r_{\theta \min }=3 \sqrt{\theta} / 2$, we find

$$
\begin{gathered}
S_{\text {rem }} \approx 3 \pi \sqrt{\theta}+2 \pi \sqrt{\theta} \ln \left(\frac{3 \sqrt{\theta}}{2}\right)+S_{0} \approx 0, \\
S_{0}=-3 \pi \sqrt{\theta}-2 \pi \sqrt{\theta} \ln \left(\frac{3 \sqrt{\theta}}{2}\right), \\
F_{\text {rem }} \approx 0+\mathcal{O}(\theta)+F_{0}, \quad F_{0}=0 .
\end{gathered}
$$

Hence, we have that the entropy and the Helmholtz free energy are zero for the remnant of the noncommutative BTZ black hole.

Now, to analyze the effect of the GUP, we consider the case where $\theta=0$ and $\alpha \neq 0$. Thus, from equation (63), we have the following contribution to specific heat:

$$
C_{\alpha}=4 \pi r_{h}\left(1-\frac{\alpha^{2}}{8 r_{h}^{2}}\right)+\cdots
$$

and the specific heat vanishes at the point $r_{h}=r_{\alpha \min }=\alpha / 2 \sqrt{2}$. Hence, the BTZ black hole with GUP becomes a remnant with a minimum mass, $M_{\alpha \min }=\alpha^{2} / 8 l^{2}$, and a temperature given by

$$
T_{\alpha \mathrm{rem}}=\frac{\left.\left(T_{\text {gup }}\right)\right|_{\theta=0}}{2}=\frac{\alpha}{8 \pi l^{2}}
$$

Moreover, the entropy and the Helmholtz free energy are zero for the remnant of the BTZ black hole with GUP with

$$
\begin{gathered}
S_{\alpha \mathrm{rem}} \approx-\pi \alpha \ln \left(\frac{\sqrt{2} \alpha}{4}\right)+S_{0} \approx 0, \quad S_{0}=\pi \alpha \ln \left(\frac{\sqrt{2} \alpha}{4}\right), \\
F_{\alpha \mathrm{rem}} \approx-(1+\sqrt{2}) \frac{\alpha^{2}}{8 l^{2}}-\sqrt{2} \alpha^{2} \ln \left(\frac{\sqrt{2} \alpha}{4}\right)+F_{0} \approx 0, \\
F_{0}=(1+\sqrt{2}) \frac{\alpha^{2}}{8 l^{2}}+\sqrt{2} \alpha^{2} \ln \left(\frac{\sqrt{2} \alpha}{4}\right) .
\end{gathered}
$$




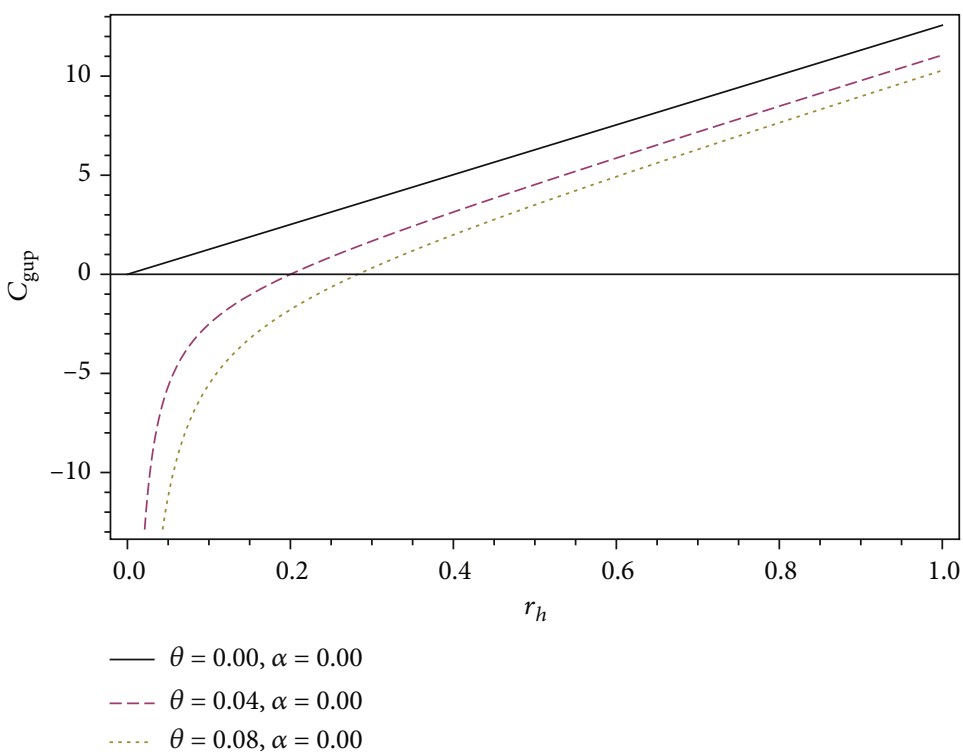

(a)

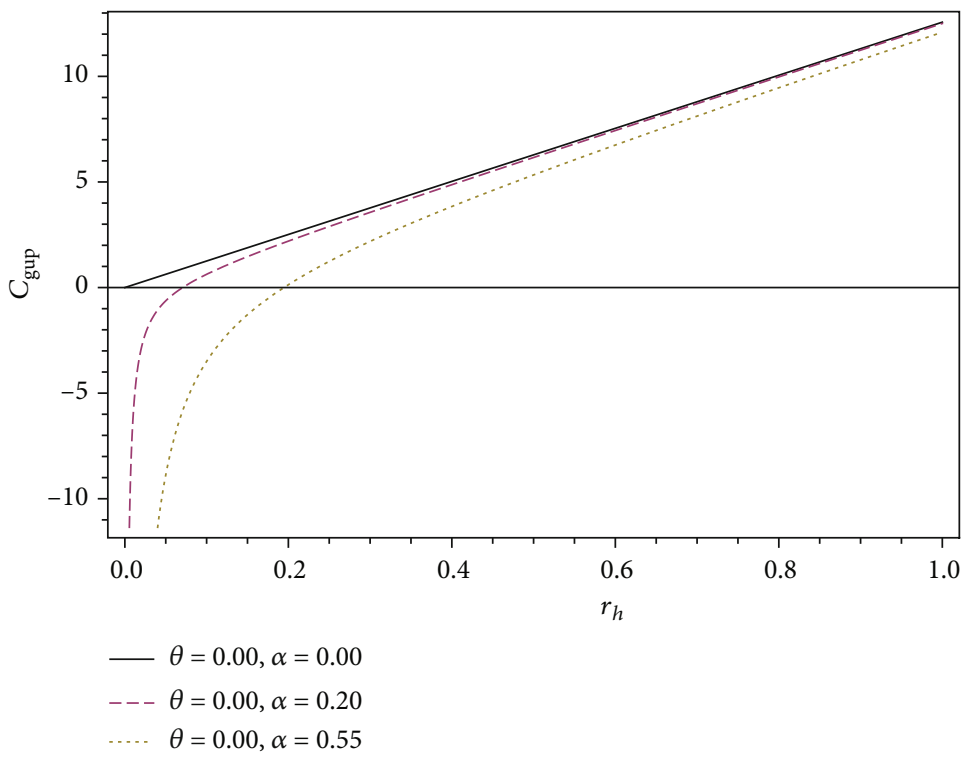

(b)

Figure 2: Specific heat capacity. (a) For $\theta \neq 0$ and $\alpha=0$, we have the result for the specific heat of the noncommutative BTZ black hole. (b) For $\theta=0$ and $\alpha \neq 0$, we have the result for the specific heat of the commutative BTZ black hole with GUP.

For $\theta \neq 0$ and $\alpha \neq 0$, we can write equation (63) as follows:

$$
C_{\text {gup }}=4 \pi \tilde{r}_{h}\left(1+\frac{\sqrt{\theta}}{2 \tilde{r}_{h}}\right)\left(1-\frac{r_{m+}}{\tilde{r}_{h}}\right)\left(1-\frac{r_{m-}}{\tilde{r}_{h}}\right)+\cdots,
$$

where

$$
r_{m \pm}=\frac{\sqrt{\theta}}{2} \pm \frac{1}{2} \sqrt{\theta+\frac{\alpha(\alpha+3 \sqrt{\theta})}{2}} .
$$

Note that for $\tilde{r}_{h}=r_{m+}$ (or $\left.r_{h}=\sqrt{\theta} / 2+r_{m+}\right)$, the specific heat vanishes. The results obtained previously are recovered when $\theta \neq 0$ and $\alpha=0$ (or $\theta=0$ and $\alpha \neq 0$ ). For the condition of forming a remnant of a noncommutative BTZ black hole, we can write the following approximations for the minimum radius:

$$
\begin{aligned}
r_{\min } & =\frac{\sqrt{\theta}}{2}+r_{m+} \\
& =\left\{\begin{array}{l}
r_{1 \min }=\frac{3 \sqrt{\theta}}{2}+\frac{3 \alpha}{8}+\mathcal{O}\left(\alpha^{2}\right), \\
r_{2 \min }=\frac{\alpha}{2 \sqrt{2}}+\frac{(3 \sqrt{2}+8)}{8} \sqrt{\theta}+\mathcal{O}(\theta) .
\end{array}\right.
\end{aligned}
$$


By applying the minimum radius $r_{\min }$, the specific heat, entropy, and Helmholtz free energy are null, and by (54) the temperature is given by

$$
\begin{aligned}
T_{\alpha \theta \text { rem }}= & \left.\left(T_{\text {gup }}\right)\right|_{r_{h}=r_{\min }}=\left(\frac{r_{\min }}{2 \pi l^{2}}-\frac{\sqrt{\theta}}{2 \pi l^{2}}\right) \\
& \cdot\left[1+\frac{\alpha}{4 r_{\min }}+\frac{\alpha}{8 r_{\min }^{2}}(\sqrt{\theta}-\alpha)-\frac{\alpha^{2} \sqrt{\theta}}{8 r_{\min }^{3}}+\cdots\right] .
\end{aligned}
$$

We can obtain approximate expressions for the temperature of the remnant of the noncommutative BTZ black hole by expanding it in $\alpha$ and $\theta$. So, by applying the minimum radii $r_{1 \min }$ and $r_{2 \mathrm{~min}}$, the temperatures are given, respectively, by

$$
\begin{aligned}
& T_{1 \text { rem }}=\left.\left(T_{\text {gup }}\right)\right|_{r_{\text {min }}=r_{1 \min }}=\frac{\sqrt{\theta}}{4 \pi l^{2}}+\frac{35 \alpha}{144 \pi l^{2}}+\mathcal{O}\left(\alpha^{2}\right), \\
& T_{2 \text { rem }}=\left.\left(T_{\text {gup }}\right)\right|_{r_{\text {min }}=r_{2 \min }}=\frac{\alpha}{8 \pi l^{2}}+\frac{\sqrt{2 \theta}}{4 \pi l^{2}}+\frac{\sqrt{\theta}}{\pi l^{2}}+\mathcal{O}(\theta) .
\end{aligned}
$$

Then, by doing $\alpha=0$ in $T_{1 \text { rem }}$, we obtain the result of (68), and for $\theta=0$ in $T_{2 \mathrm{rem}}$, we recover the result of (71).

Now in order to verify the stability of the black hole, we show in Figures 1 and 2 the specific heat behavior. In Figure 1, we show that the specific heat is positive for $\theta=0.001$ and $\alpha=0.1$, and so the noncommutative BTZ black hole with GUP is stable. In addition, we observed that the specific heat vanishes to a critical radius. Furthermore, for $\theta=0.03$ and $\alpha=0.1$, one achieves two points where the specific heat vanishes, with an unphysical region in between.

In Figure 2(a), we verify the behavior of the specific heat for $\theta \neq 0$ and $\alpha=0$, and in Figure 2(b) for $\theta=0$ and $\alpha \neq 0$. Note that the specific heat vanishes before entering into an unphysical zone. The BTZ black hole decreases its size until it achieves a critical radius where it ceases to evaporate and becomes a remnant of the noncommutative BTZ black hole.

\section{Conclusions}

In summary, we have considered the metric of a noncommutative BTZ black hole implemented via the Lorentzian mass distribution. Thus, applying the Hamilton-Jacobi approach and the WKB approximation, we have obtained noncommutative corrections to the Hawking temperature and entropy. In addition, we have found a logarithmic correction to the entropy of the BTZ black hole due to the effect of noncommutativity. We also have verified the stability of the BTZ black hole by calculating the specific heat capacity and have shown that the noncommutative BTZ black hole becomes a remnant with a minimum mass $M_{\theta \text { min }}=9 \theta / 4 l^{2}$. Therefore, the contribution of the noncommutative corrections introduces a GUP effect. We also investigated the effect of GUP by calculating the Hawking temperature and entropy of the noncommutative BTZ black hole. Due to the effect of noncommutativity and GUP, we have found logarithmic corrections for the entropy of the BTZ black hole, in the form of $S_{\text {gup }} \sim S+\left(c_{1}+c_{2}\right) \ln S+\cdots$, where the "species" $c_{i}=(-\alpha, \theta)$ are essentially related to each corresponding parameter of correction.

\section{Data Availability}

No data were used to support this study.

\section{Conflicts of Interest}

The authors declare that they have no conflicts of interest.

\section{Acknowledgments}

We would like to thank CNPq, CAPES, and CNPq/PRONEX/FAPESQ-PB (Grant nos. 165/2018 and 015/2019), for partial financial support. MAA, FAB, and EP acknowledge support from CNPq (Grant nos. 306962/2018-7, 433980/ 2018-4, 312104/2018-9, and 304852/2017-1).

\section{References}

[1] S. Carlip, "The $(2+1)$-dimensional black hole," Classical and Quantum Gravity, vol. 12, no. 12, pp. 2853-2879, 1995.

[2] J. M. Maldacena, "The large $N$ limit of superconformal field theories and supergravity," Advances in Theoretical and Mathematical Physics, vol. 2, no. 2, pp. 231-252, 1998.

[3] M. Banados, C. Teitelboim, and J. Zanelli, "Black hole in threedimensional spacetime," Physical Review Letters, vol. 69, no. 13, pp. 1849-1851, 1992.

[4] K. C. K. Chan and R. B. Mann, "Spinning black holes in $(2+1)$ dimensional string and dilaton gravity," Physics Letters B, vol. 371, no. 3-4, pp. 199-205, 1996.

[5] C. Martinez and J. Zanelli, "Conformally dressed black hole in $2+1$ dimensions," Physical Review D, vol. 54, no. 6, pp. 38303833, 1996.

[6] K. C. K. Chan, "Modifications of the BTZ black hole by a dilaton and scalar," Physical Review D, vol. 55, no. 6, pp. 35643574, 1997.

[7] P. Nicolini, "Noncommutative black holes, the final appeal to quantum gravity: a review," International Journal of Modern Physics, vol. 24, pp. 1229-1308, 2012.

[8] P. Nicolini, A. Smailagic, and E. Spallucci, "Noncommutative geometry inspired Schwarzschild black hole," Physics Letters $B$, vol. 632, no. 4, pp. 547-551, 2006.

[9] K. Nozari and S. H. Mehdipour, "Hawking radiation as quantum tunneling from a noncommutative Schwarzschild black hole," Classical and Quantum Gravity, vol. 25, no. 17, p. 175015, 2008.

[10] M. Bañados, O. Chandía, N. E. Grandi, F. A. Schaposnik, and G. A. Silva, "Chern-Simons formulation of noncommutative gravity in three dimensions," Physical Review D, vol. 64, no. 8, article $084012,2001$.

[11] H.-C. Kim, M.-I. Park, C. Rim, and J. H. Yee, "Smeared BTZ black hole from space noncommutativity," Journal of High Energy Physics, vol. 10, p. 60, 2008. 
[12] E. Chang-Young, D. Lee, and Y. Lee, "The noncommutative BTZ black hole in polar coordinates," Classical and Quantum Gravity, vol. 26, article 185001, 2009.

[13] R. Jahangir and K. Saifullah, "Thermodynamics of noncommutative BTZ black hole," https://arxiv.org/abs/1101.6073.

[14] J. Liang and B. Liu, "Thermodynamics of noncommutative geometry inspired BTZ black hole based on Lorentzian smeared mass distribution," EPL, vol. 100, no. 3, p. 30001, 2012.

[15] M. A. Anacleto, F. A. Brito, and E. Passos, "Gravitational Aharonov-Bohm effect due to noncommutative BTZ black hole," Physics Letters B, vol. 743, pp. 184-188, 2015.

[16] M. A. Anacleto, F. A. Brito, J. A. V. Campos, and E. Passos, "Absorption and scattering of a noncommutative black hole," Physics Letters B, vol. 803, p. 135334, 2020.

[17] F. Rahaman, P. K. F. Kuhfittig, B. C. Bhui, M. Rahaman, S. Ray, and U. F. Mondal, "BTZ black holes inspired by noncommutative geometry," Physical Review D, vol. 87, no. 8, article 084014, 2013.

[18] J. Sadeghi and V. R. Shajiee, "Effective potential in noncommutative BTZ black hole," International Journal of Theoretical Physics, vol. 55, no. 2, pp. 892-900, 2016.

[19] M. A. Anacleto, F. A. Brito, A. G. Cavalcanti, E. Passos, and J. Spinelly, "Quantum correction to the entropy of noncommutative BTZ black hole," General Relativity and Gravitation, vol. 50, no. 2, 2018.

[20] S. Kawamoto, K. Nagasaki, and W. Y. Wen, "Charged rotating BTZ black holes in noncommutative spaces and torsion gravity," Progress of Theoretical and Experimental Physics, vol. 2018, no. 4, article 043E01, 2018.

[21] S. Das and E. C. Vagenas, "Universality of quantum gravity corrections," Physical Review Letters, vol. 101, no. 22, article 221301, 2008.

[22] S. Das and E. C. Vagenas, "Phenomenological implications of the generalized uncertainty principle. This paper was presented at the Theory CANADA 4 conference, held at Centre de recherches mathématiques, Montréal, Québec, Canada on 4-7 June 2008," Canadian Journal of Physics, vol. 87, no. 3, pp. 233-240, 2009.

[23] A. F. Ali, S. Das, and E. C. Vagenas, "Proposal for testing quantum gravity in the lab," Physical Review D, vol. 84, article 044013, 2011.

[24] M. A. Anacleto, F. A. Brito, C. V. Garcia, G. C. Luna, and E. Passos, "Quantum-corrected rotating acoustic black holes in Lorentz-violating background," Physical Review D, vol. 100, no. 10, article 105005, 2019.

[25] G. Gecim and Y. Sucu, "The GUP effect on tunneling of massive vector bosons from the $2+1$ dimensional black hole," Advances in High Energy Physics, vol. 2018, Article ID 7031767, 8 pages, 2018.

[26] G. Gecim and Y. Sucu, "Massive vector bosons tunnelled from the $(2+1)$-dimensional black holes," The European Physical Journal Plus, vol. 132, no. 3, p. 105, 2017.

[27] M. A. Anacleto, F. A. Brito, and E. Passos, "Quantum-corrected self-dual black hole entropy in tunneling formalism with GUP," Physics Letters B, vol. 749, pp. 181-186, 2015.

[28] M. A. Anacleto, D. Bazeia, F. A. Brito, and J. C. Mota-Silva, "Quantum-corrected two-dimensional Horava-Lifshitz black hole entropy," Advances in High Energy Physics, vol. 2016, Article ID 8465759, 11 pages, 2016.

[29] M. A. Anacleto, F. A. Brito, E. Passos, and W. P. Santos, “The entropy of the noncommutative acoustic black hole based on generalized uncertainty principle," Physics Letters B, vol. 737, pp. 6-11, 2014.

[30] C. A. S. Silva and F. A. Brito, "Quantum tunneling radiation from self-dual black holes," Physics Letters B, vol. 725, no. 45, pp. 456-462, 2013.

[31] T. I. Singh, Y. K. Meitei, and I. A. Meitei, "Effect of GUP on Hawking radiation of BTZ black hole," International Journal of Modern Physics A, vol. 35, no. 5, article 2050018, 2020.

[32] G. Gecim and Y. Sucu, "The GUP effect on Hawking radiation of the $2+1$ dimensional black hole," Physics Letters B, vol. 773, pp. 391-394, 2017.

[33] G. Gecim and Y. Sucu, "Quantum gravity effect on the tunneling particles from 2+1-dimensional new-type black hole," Advances in High Energy Physics, vol. 2018, Article ID 8728564, 7 pages, 2018.

[34] G. Gecim and Y. Sucu, "Quantum gravity effect on the Hawking radiation of charged rotating BTZ black hole," General Relativity and Gravitation, vol. 50, no. 12, 2018.

[35] I. A. Meitei, T. I. Singh, S. G. Devi, N. P. Devi, and K. Y. Singh, "Quantum gravity effects on scalar particle tunneling from rotating BTZ black hole," International Journal of Modern Physics A: Particles and Fields; Gravitation; Cosmology; Nuclear Physics, vol. 33, no. 12, p. 1850070, 2018.

[36] J. Sadeghi and V. Reza Shajiee, "Quantum tunneling from the charged non-rotating BTZ black hole with GUP," The European Physical Journal Plus, vol. 132, no. 3, 2017.

[37] E. Maghsoodi, H. Hassanabadi, and W. Sang Chung, "Effect of the new extended uncertainty principle on black hole thermodynamics," EPL, vol. 129, no. 5, p. 59001, 2020.

[38] M. Visser, "Area products for stationary black hole horizons," Physical Review D, vol. 88, no. 4, article 044014, 2013.

[39] A. F. Ali, S. Das, and E. C. Vagenas, "Discreteness of space from the generalized uncertainty principle," Physics Letters $B$, vol. 678, no. 5, pp. 497-499, 2009.

[40] A. N. Tawfik and A. M. Diab, "Generalized uncertainty principle: approaches and applications," International Journal of Modern Physics D, vol. 23, article 1430025, 2014.

[41] A. N. Tawfik and E. A. E. Dahab, "Corrections to entropy and thermodynamics of charged black hole using generalized uncertainty principle," International Journal of Modern Physics A, vol. 30, no. 9, article 1550030, 2015.

[42] S. Gangopadhyay, A. Dutta, and M. Faizal, "Constraints on the generalized uncertainty principle from black-hole thermodynamics," EPL (Europhysics Letters), vol. 112, no. 2, article 20006, 2015.

[43] J. Magueijo and L. Smolin, "Lorentz invariance with an invariant energy scale," Physical Review Letters, vol. 88, 2002.

[44] J. L. Cortés and J. Gamboa, "Quantum uncertainty in doubly special relativity," Physical Review D, vol. 71, 2005.

[45] F. Hammad, " $f(R)$-modified gravity, Wald entropy, and the generalized uncertainty principle," Physical Review D, vol. 92, 2015.

[46] A. Kempf, G. Mangano, and R. B. Mann, "Hilbert space representation of the minimal length uncertainty relation," Physical Review D, vol. 52, article 1108, 1995.

[47] S. Gangopadhyay, A. Dutta, and A. Saha, "Generalized uncertainty principle and black hole thermodynamics," General Relativity and Gravitation, vol. 46, article 1661, 2014.

[48] E. Carlen and R. Vilela Mendes, "Non-commutative spacetime and the uncertainty principle," Physics Letters A, vol. 290, pp. 109-114, 2001. 
[49] C. Bastos, O. Bertolami, N. C. Dias, and J. N. Prata, "Noncanonical phase-space noncommutativity and the KantowskiSachs singularity for black holes," Physical Review D, vol. 84, article 024005, 2011.

[50] B. Pourhassan, A. Övgün, and I. Sakalli, "PV criticality of Achucarro-Ortiz black hole in the presence of higher-order quantum and GUP corrections," International Journal of Geometric Methods in Modern Physics, vol. 17, no. 10, article 2050156, 2020.

[51] J. Sadeghi, B. Pourhassan, and F. Rahimi, "Logarithmic corrections of charged hairy black holes in $(2+1)$ dimensions," Canadian Journal of Physics, vol. 92, no. 12, article 1638, 2014.

[52] J. Sadeghi, B. Pourhassan, and M. Rostami, " $P-V$ criticality of logarithm-corrected dyonic charged AdS black holes," Physical Review D, vol. 94, no. 6, article 064006, 2016.

[53] B. Pourhassan, M. Faizal, and S. A. Ketabi, "Logarithmic correction of the BTZ black hole and adaptive model of graphene," International Journal of Modern Physics D, vol. 27, no. 12, article 1850118, 2018.

[54] S. Upadhyay and B. Pourhassan, "Logarithmic-corrected van der Waals black holes in higher-dimensional AdS space," Progress of Theoretical and Experimental Physics, vol. 2019, article 013B03, no. 1, 2019. 